\title{
AN APPLICATION OF THE METHOD OF PARAMETERS TO LINEAR PARTIAL DIFFERENTIAL EQUATIONS*
}

BY F. H. MILLER

1. Introduction. The method of variation of parameters provides, as is well known, an elegant means of finding the complete primitive of a linear ordinary differential equation whose complementary function is given. In this paper it is shown that the method is applicable also to certain linear partial differential equations of the second order and that the results so obtained are interconnected with the familiar Laplace transformation.

In what follows we shall understand that the functions of $x$ and $y$ considered are of class $C^{\prime \prime}$ in a domain of definition $K$, where $C^{\prime \prime}$ is the class of functions which are continuous together with their first and second derivatives. We shall suppose that the complementary function of our differential equation is of the form $F(\alpha)+G(\beta), \alpha$ and $\beta$ being known distinct expressions in $x$ and $y$, and $F$ and $G$ arbitrary functions, in $C^{\prime \prime}$. It may be readily verified that any differential equation having such a complementary function will be of the type

$$
\left(R D^{2}+S D D^{\prime}+T D^{\prime 2}+P D+Q D^{\prime}\right) z=V,
$$

where $R, S, \cdots, V$ are functions of $x$ and $y$ alone and $D \equiv$ $\partial / \partial x, D^{\prime} \equiv \partial / \partial y$. Not every equation conforming to (1), however, will have $F(\alpha)+G(\beta)$ as complementary function; a criterion that may be easily applied arises from the following conditions, $\dagger$ necessary and sufficient in class $C^{\prime \prime}$ :

$$
\begin{aligned}
R \alpha_{x}{ }^{2}+S \alpha_{x} \alpha_{y}+T \alpha_{y}{ }^{2} & =0 \\
R \beta_{x}{ }^{2}+S \beta_{x} \beta_{y}+T \beta_{y}{ }^{2} & =0 \\
R \alpha_{x x}+S \alpha_{x y}+T \alpha_{y y}+P \alpha_{x}+Q \alpha_{y} & =0 \\
R \beta_{x x}+S \beta_{x y}+T \beta_{y y}+P \beta_{x}+Q \beta_{y} & =0
\end{aligned}
$$

* Presented to the Society, October 26, 1929.

$\dagger$ It should be noticed that equations (2a)-(2d) place symmetrical hypotheses on $\alpha$ and $\beta$; this symmetry will be made use of later in the discussion. 
These relations must be satisfied by $\alpha$ and $\beta$ identically in $x$ and $y$; for, if we substitute $z=F(\alpha)+G(\beta)$ in the homogeneous equation obtained from (1) by replacing $V$ by zero, it follows that since $F$ and $G$ are arbitrary, the coefficients of their first and second derivatives must vanish identically in $K$, whence we have the above relations.

We further restrict the domain $K$ by assuming, throughout our work, $S^{2}-4 R T \neq 0, J=\alpha_{x} \beta_{y}-\alpha_{y} \beta_{x} \neq 0$, in $K$. In $\$ 2$ we confine our attention to the case in which the first partial derivatives of $\alpha$ and $\beta$ are all different from zero in $K$, and in $\S 3$ we consider the special cases in which one or more of these derivatives vanish identically in $K$; it will also be found necessary in each of these two discussions to assume $K$ restricted so that solutions of certain associated ordinary differential equations needed in the process be obtainable.*

In the last section we shall indicate the relation between our previously derived results and the process of effecting a Laplace transformation.

2. The General Case. The criterion mentioned in the introduction for the determination of those equations having complementary functions $F(\alpha)+G(\beta)$, when the first partial derivatives of $\alpha$ and $\beta$ are different from zero in $K$, is indicated by the form of equations (2a)-(2d). We obtain the roots of the quadratic $R \theta^{2}+S \theta+T=0$, whose discriminant is by hypothesis different from zero in $K$, and denote them by $\alpha_{x} / \alpha_{y}$ and $\beta_{x} / \beta_{y}$ in accordance with equations (2a) and (2b); $\dagger$ the functions $\alpha$ and $\beta$ so determined must then, by (2c) and (2d), themselves satisfy the homogeneous equation corresponding to (1).

Supposing this test fulfilled, and that we are in possession of the complementary function, we assume the general solution of (1) to be

$$
z=\alpha u+\beta v+w,
$$

where $u, v, w$ are unknown functions of $x$ and $y$ in $C^{\prime \prime}$ to be employed as parameters. Consequently, taking

\footnotetext{
* The equations referred to are (11), (14), and the corresponding ones of $\$ 3$.

$\dagger$ The quadratic in $\theta$ shows us that, apart from a possible common factor, $R=\alpha_{y} \beta_{y}, S=-\left(\alpha_{x} \beta_{y}+\alpha_{y} \beta_{x}\right), T=\alpha_{x} \beta_{x}$; thus $R$ and $T$ are in the present case certainly different from zero in $K$.
} 


$$
\begin{aligned}
& \alpha u_{x}+\beta v_{x}+w_{x}=0, \\
& \alpha u_{y}+\beta v_{y}+w_{y}=0,
\end{aligned}
$$

as the two additional conditions on our parameters, we find

$$
\left\{\begin{aligned}
D z & =\alpha_{x} u+\beta_{x v}, \quad D^{\prime} z=\alpha_{y} u+\beta_{y} v, \\
D^{2} z & =\alpha_{x} u_{x}+\alpha_{x x} u+\beta_{x} v_{x}+\beta_{x x} v, \\
D D^{\prime} z & =\alpha_{y} u_{x}+\alpha_{x y} u+\beta_{y} v_{x}+\beta_{x y} v, \\
D^{\prime} D z & =\alpha_{x} u_{y}+\alpha_{x y} u+\beta_{x} v_{y}+\beta_{x y} v, \\
D^{\prime 2} z & =\alpha_{y} u_{y}+\alpha_{y y} u+\beta_{y} v_{y}+\beta_{y y} v .
\end{aligned}\right.
$$

Since $D D^{\prime} z$ must be identical with $D^{\prime} D z$, ${ }^{*}$ it follows that

$$
\alpha_{y} u_{x}-\alpha_{x} u_{y}=-\beta_{y} v_{x}+\beta_{x} v_{y} .
$$

From (4) and (5) we get $\alpha d u+\beta d v+d w=0$; combining this with $d z$ found from (3), we obtain

$$
d z=u d \alpha+v d \beta=\left(u \alpha_{x}+v \beta_{x}\right) d x+\left(u \alpha_{y}+v \beta_{y}\right) d y .
$$

By virtue of relation (7), the last member of (8) is seen to be a total differential. Hence it is necessary merely to determine $u$ and $v$, insert their values in (8), and integrate.

Substituting from (6) in the given equation (1) yields, after reduction by means of equations (2d) and (2c),

(9) $\left(R \alpha_{x}+S \alpha_{y}\right) u_{x}+T \alpha_{y} u_{y}+\left(R \beta_{x}+S \beta_{y}\right) v_{x}+T \beta_{y} v_{y}=V$.

Now, from (2b), $-\left(R \beta_{x}+S \beta_{y}\right) / \beta_{y}=T \beta_{y} / \beta_{x}$; making use of this after multiplying equation (7) throughout by $-\left(R \beta_{x}+S \beta_{y}\right) / \beta_{y}$, we have

$$
\begin{aligned}
-\alpha_{y}\left(R \beta_{x}+S \beta_{y}\right) u_{x}+\alpha_{x}\left(R \beta_{x}+\right. & \left.S \beta_{y}\right) u_{y} \\
& =\beta_{y}\left(R \beta_{x}+S \beta_{y}\right) v_{x}+T \beta_{y}{ }^{2} v_{y} .
\end{aligned}
$$

Eliminating $v_{x}$ and $v_{y}$ between this equation and (9), we obtain a. linear partial differential equation of first order in $u$, the subsidiary equations of which are

$$
d x /(R J)=d y /\left(R \alpha_{x} \beta_{x}+S \alpha_{x} \beta_{y}+T \alpha_{y} \beta_{y}\right)=d u /\left(\beta_{y} V\right) .
$$

As an immediate consequence of (2a), we get from (10), $\alpha=c_{1}$,

\footnotetext{
* For $z$ as given by (3) is by hypothesis in $C^{\prime \prime}$.
} 
a constant. Solving $\alpha=c_{1}$ for $y^{*}$ in terms of $x$ and $c_{1}$, and inserting this value of $y$ in (10), there is obtained

$$
\frac{d u}{d x}=\frac{\beta_{y} V}{R J} .
$$

Integrating (11) and replacing $c_{1}$ by $\alpha$ in the result gives us $u=\phi(x, y)+$ const. Consequently we have

$$
u=\phi(x, y)+F_{1}(\alpha),
$$

where $F_{1}$ is an arbitrary function in $C^{\prime \prime}$.

The function $v$ is found in precisely the same manner, $\dagger$ the equation determining it being

$$
-R J v_{x}+\left(R \alpha_{x} \beta_{x}+S \alpha_{y} \beta_{x}+T \alpha_{y} \beta_{y}\right) v_{y}=\alpha_{y} V .
$$

It follows in this case that the equations subsidiary to (13) give us, by (2b), $\beta=c_{2}$, and therefore

$$
\frac{d v}{d x}=-\frac{\alpha_{y} v}{R J}
$$

where again $y$ is supposed replaced by its value from $\beta=c_{2}$. Hence there is found

$$
v=\psi(x, y)+G_{1}(\beta) .
$$

Substituting from (12) and (15) in (8), we get finally

$$
d z=\left(\phi \alpha_{x}+\psi \beta_{x}\right) d x+\left(\phi \alpha_{y}+\psi \beta_{y}\right) d y+F_{1}(\alpha) d \alpha+G_{1}(\beta) d \beta,
$$

and

$$
z=\int\left[\left(\phi \alpha_{x}+\psi \beta_{x}\right) d x+\left(\phi \alpha_{y}+\psi \beta_{y}\right) d y\right]+F(\alpha)+G(\beta),
$$

the first member on the right representing the desired particular integral.

3. Special Cases. The existence of what we shall call special or exceptional cases, in which one or more first derivatives of $\alpha$ and $\beta$ vanish identically in $K$, is easily recognized from the form of equation (1). For equations (2a) and (2b) show that

* This is possible since $\alpha_{y} \neq 0$ in $K$.

$\dagger$ It is here that the symmetrical hypotheses on $\alpha$ and $\beta$ play a part. The function $u$ having been found, $v$ might also be obtained from (7); however, because of symmetry, and for use in $\$ 4$, equation (13) is preferable. 
the vanishing of a derivative of $\alpha$ or $\beta$ entails the vanishing of $R$ or $T$, so that if one of these derivatives is identically zero in $K$ the term in $D^{2} z$ or $D^{\prime 2} z$ will be absent from the differential equation. In such event also, the arguments $\alpha$ and $\beta$ are found without difficulty, ${ }^{*}$ and we may proceed to the evaluation of the particular integral.

(a) First suppose that instead of having $\alpha_{x} \neq 0$ we now have $\alpha_{x} \equiv 0$ in $K$, while all other restrictions previously imposed on $K$ and the differential equation (1) are retained. Then, by (2a), $T$ must be identically zero in $K$. Consequently equations (7) and (9) reduce to

$$
\begin{gathered}
\alpha_{y} u_{x}=-\beta_{y} v_{x}+\beta_{x} v_{y}, \\
S \alpha_{y} u_{x}+\left(R \beta_{x}+S \beta_{y}\right) v_{x}=V,
\end{gathered}
$$

respectively. We may evidently combine these so as to obtain a partial differential equation of first order determining $v$, and knowing this function, we may find $u$ from (7a). The case in which $\beta_{x} \equiv 0$ instead of $\alpha_{x} \equiv 0$ is precisely the same except that $\alpha$ and $\beta$, and $u$ and $v$, are interchanged in (7a) and (9a).

(b) If, other conditions being the same, we next suppose that $\alpha_{y}$ in place of $\alpha_{x}$ is identically zero in $K$, equation (2a) shows us that $R$ must vanish, and therefore equations (7) and (9) become

$$
\begin{gathered}
-\alpha_{x} u_{y}=-\beta_{y} v_{x}+\beta_{x} v_{y}, \\
S \beta_{y} v_{x}+T \beta_{y} v_{y}=V .
\end{gathered}
$$

Equation (9b) determines $v$ directly and (7b) then gives us $u$. Again, the case in which $\beta_{y} \equiv 0$ in place of $\alpha_{y} \equiv 0$ is entirely similar.

(c) Finally, suppose the conditions altered by having $\alpha_{x}$ and $\beta_{y}$ both identically zero in $K$. Then

$$
\begin{aligned}
\alpha_{y} u_{x} & =\beta_{x} v_{y}, \\
S \alpha_{y} u_{x} & =V .
\end{aligned}
$$

The functions $u$ and $v$ are readily obtainable from these relations. If $\alpha_{y}$ and $\beta_{x}$ vanish identically in $K$, a like pair of equations serves to fix $u$ and $v$.

* See, for example, Forsyth's Theory of Differential Equations, 1906, vol. vi, p. 32, cases iii-v. 
Obviously both first derivatives of $\alpha$ (or of $\beta$ ) cannot vanish identically in $K$ simultaneously, nor can we have $\alpha_{x}=\beta_{x}=0$ (or $\alpha_{y}=\beta_{y}=0$ ) identically in $K$, since by hypothesis the jacobian $J$ does not vanish in $K$. Thus, the exceptional cases are all amenable to the method described in the preceding section.

4. The Laplace Transformation. If we take $\alpha$ and $\beta$ as new independent variables, equation (1) reduces to

$$
\partial^{2} z / \partial \alpha \partial \beta=-\alpha_{y} \beta_{y} V /\left(R J^{2}\right),
$$

assuming the conditions of $\$ 2$ fulfilled. The right-hand member of (17) may also be expressed as $-\alpha_{x} \beta_{x} V /\left(T J^{2}\right)$; cases (a) and (b) of the preceding section are readily shown to submit to one or the other of these two modes of expression, while (c) obviates the necessity of any transformation, since (1) must then be simply $S D D^{\prime} z=V$ by (2a)-(2d). Now if $\alpha=\alpha(x, y)$ and $\beta=\beta(x, y)$ can actually be solved* for $x$ and $y$ in terms of $\alpha$ and $\beta$, substitution of these values in the right member of (17) followed by two simple integrations yields the general solution. We shall call such a procedure a "direct" application of Laplace's transformation. In some cases, however, it is not possible to effect this substitution, as the following example illustrates.

Let there be given the differential equation $\left[x(1-x) D^{2}-y\left(1-x^{2}\right) D D^{\prime}+y^{2}(1-x) D^{\prime 2}+D-x y D^{\prime}\right] z=1$, and suppose the domain $K$ to consist of any region not including those points for which $x=0$ or 1 or $y=0$. Using the relations (2a)-(2d), we find that $F(x y)+G\left(y e^{x}\right)$ is the complementary function. Now it is a practical impossibility to solve the equations $\alpha=x y, \beta=y e^{x}$ simultaneously for $x$ and $y$, and consequently Laplace's transformation cannot be directly applied. But equations (11) and (14) are here $d u / d x=1 /\left[c_{1}(1-x)^{2}\right]$, $d v / d x=-1 /\left[c_{2}(1-x)^{2}\right]$, so that $\phi=1 /[x y(1-x)], \psi=-1 /\left[y e^{x}(1-x)\right]$. Substitution in (16) therefore gives as the general solution $z=\log x+F(x y)+G\left(y e^{x}\right)$.

Thus, when a given differential equation of the type here considered does not lend itself to a direct application of (17), we may nevertheless obtain the general solution from equation

* Of course, this can theoretically always be accomplished, aside from algebraic difficulties, under our hypotheses. 
(16). I am indebted to H. W. Reddick for pointing out to me that (16) may also be viewed as a consequence of equation (17); that is, the result obtained from the standpoint of the parametric method is also intimately connected with the Laplace transformation. Since

$$
\alpha_{x}=J y_{\beta}, \alpha_{y}=-J x_{\beta}, \beta_{x}=-J y_{\alpha}, \beta_{y}=J x_{\alpha},
$$

equation (17) gives us*

$$
\begin{aligned}
& \frac{\partial z}{\partial \alpha}=\int_{\beta} \frac{\beta_{y} V}{R J} \frac{\partial x}{\partial \beta} d \beta+F_{1}(\alpha), \\
& \frac{\partial z}{\partial \beta}=-\int_{\alpha} \frac{\alpha_{y} V}{R J} \frac{\partial x}{\partial \alpha} d \alpha+G_{1}(\beta),
\end{aligned}
$$

where the subscript on an integral sign denotes the variable with respect to which the integration is to be performed. But when we integrate with respect to $\beta, x_{\beta} d \beta$ is merely $d x$; likewise, in the second of equations (19), $x_{\alpha} d \alpha$ will be $d x$. Hence the above two integrals are simply the expressions denoted by $\phi$ and $\psi$ respectively, and therefore

$$
\begin{aligned}
z & =\int\left(z_{\alpha} d \alpha+z_{\beta} d \beta\right)+F(\alpha)+G(\beta) \\
& =\int(\phi d \alpha+\psi d \beta)+F(\alpha)+G(\beta),
\end{aligned}
$$

which is our equation (16).

Theoretically, of course, the solution of equation (1) is expressed by writing

$$
z=\int_{\beta} \int_{\alpha} \frac{V}{R} \frac{\partial x}{\partial \alpha} \frac{\partial x}{\partial \beta} d \alpha d \beta+F(\alpha)+G(\beta)
$$

which follows directly from (17); our purpose here has been to indicate the manner in which the particular integral of (20) may be explicitly determined. Although, as we have seen, our method of attack may be viewed in connection with a Laplace transformation, it is interesting to observe that these results also follow from the familiar idea of variation of parameters.

\section{Columbia Universtty}

* We are again supposing the hypotheses of $\$ 2$ to be satisfied. In the exceptional cases (17) may be taken in another form. as previously stated: the details need not be considered anew. 